\title{
A Prospective Study to Understand the Risk Factors for Anastomotic Leak in Small Bowel Anastomosis
}

\author{
Lavanya N. R. ${ }^{1}$, Janardhana T. ${ }^{2}$ \\ ${ }^{1}$ Department of Surgery, KMCH, Palakkad, Kerala, India. \\ ${ }^{2}$ Department of Surgery, KMCH, Palakkad, Kerala, India.
}

\section{ABSTRACT}

\section{BACKGROUND}

Small intestinal anastomosis is a common GI procedure. Anastomotic leaks increase morbidity, mortality and hospital stay in patients undergoing bowel anastomosis. Understanding the risk factors associated with anastomotic leak not only helps to reduce the incidence of leaks but also to identify the patients who are candidates for creation of stomas. The aim of the study is to define the risk factors associated with anastomotic leak following small bowel anastomosis.

\section{METHODS}

A total of 74 patients who underwent small bowel anastomosis in our study period between September 2016 and December 2019 in Karuna Medical College, Palakkad, were prospectively followed up and divided into two groups, leak and non-leak group depending on occurrence of anastomotic leak. Many host and disease related factors known to influence the outcome of anastomosis were recorded in both groups and compared.

\section{RESULTS}

Out of 74 patients, 40 were males and 34 females. 10 patients were aged more than 60 years. 26 of them had perforation and peritoneal contamination. Out of 74, 67 were emergency surgeries and 7 were elective surgeries. 14 patients were anaemic, 19 were hyponatraemic and 12 were hypoalbunemic. In total, 13 patients developed anastomotic leak. Overall mortality and morbidity was $6.7 \%$ (5 patients) and morbidity was $40.5 \%$ (30 patients). Mortality in anastomotic leak group was $23 \%$ (3 patients).

\section{CONCLUSIONS}

Hypoalbuminemia (P value- 0.016) and presence of peritoneal contamination ( $\mathrm{P}$ value $=0.004$ ) were found to significantly increase the risk of anastomotic leak in the present study. In the presence these risk factors, it is better to consider the patient for diverting stoma in emergency setting and consider improving patient nutritional status in elective setting.

\section{KEY WORDS}

Small Bowel Resection and Anastomosis, Anastomotic Leak, Hypoalbuminemia, Peritoneal Contamination
Corresponding Author:

Dr. Janardhana $T$.,

Assistant Professor

Department of Surgery,

KMCH, Palakkad,

Kerala, India.

E-mail: drjohn.johnny@gmail.com

DOI: $10.14260 /$ jemds/2020/338

Financial or Other Competing Interests: None.

How to Cite This Article:

Lavanya NR, Janardhana T. A prospective study to understand the risk factors for anastomotic leak in small bowel anastomosis. J. Evolution Med. Dent. Sci. 2020;9(19):1549-1553, DOI:
Submission 26-03-2020,

Peer Review 26-04-2020,

Acceptance 29-04-2020,

Published 11-05-2020. 


\section{BACKGROUND}

The word anastomosis comes from the Greek word ' $\alpha v \alpha$ ', meaning without, and ' $\sigma \tau \mu^{\alpha} \alpha$ ' a mouth, i.e. when a tubular viscus (bowel) or vessel (mostly arteries) is joined after resection or bypass without exteriorisation with a stoma. ${ }^{1}$ Before $19^{\text {th }}$ century, intestinal surgery was limited to creation of stomas, or closure of simple lacerations. In 1926, Lembert described the sero-muscular suture technique for bowel anastomosis. Kocher advocated a two layer anastomosis, $1^{\text {st }} \mathrm{a}$ continuous all-layer suture using catgut, then an inverting interrupted or continuous seromuscular layer suture using silk, which became the mainstay of bowel anastomosis for many years. Later Matheson favoured the use of one layer extra-mucosal anastomosis as it was felt to cause the least tissue necrosis or luminal narrowing. It is of at most importance to include sub-mucosal layer in the extra-mucosal suture, as this layer has high collagen content and is the most stable suture layer in all section of the gastro-intestinal tract. ${ }^{1}$ Resection and anastomosis of small bowel is one of the common general surgical procedure in the present clinical practice, especially in emergency setting. Anastomotic leaks are one of the dreaded complications of bowel surgery owing to their significant morbidity and mortality. ${ }^{2,3}$ Anastomotic leak can be defined as any defect in suture or staple line of the bowel anastomosis, leading to communication between the intra and extra luminal compartments. ${ }^{4}$ Anastomotic leaks may result in peritonitis, fistulization, intra-abdominal abscess and other fatal septic complications. ${ }^{4}$ Enteric fistulas especially are extremely complex and troublesome presentation of anastomotic leak. ${ }^{5}$ Entero-cutaneous fistulas results in loss of electrolytes and protein rich fluids. Small bowel fistulas on an average results in the loss of about 3500 $\mathrm{ml}$ of fluid, $450 \mathrm{mEq}$ of sodium, $50 \mathrm{mEq}$ of potassium, $100 \mathrm{mEq}$ of bicarbonates, and $400 \mathrm{mEq}$ of chlorides per day. ${ }^{5}$ Edmunds et al. recognized the role of sepsis, malnutrition and electrolyte abnormalities in contributing to the morbidity and mortality of entero-cutaneous fistulas. ${ }^{6}$ Mortality following anastomotic leak remains high between $10-30 \% .5$ Anastomotic healing follows the same principles of wound healing, and hence risk factors for developing an anastomotic leak are identical to those that predict wound dehiscence. Systemic risk factors include age, malnutrition, vitamin deficiencies, diabetes, smoking, inflammatory bowel disease, previous radiation/chemotherapy and anaemia. Local risk factors include tension, poor blood flow, hypotension, radiation, and peritoneal contamination. ${ }^{2}$ There are a lot of studies conducted on factors influencing colorectal anastomosis, ${ }^{3,7}$ but there is paucity of studies conducted on factors influencing small bowel anastomosis

Factors responsible for anastomotic leaks can be divided into host related factors and those related to surgical technique. However, with adequate supervision, there is little difference between the outcomes of anastomoses performed by trainees and those performed by established surgeons. ${ }^{8}$ Hence, the host related factors influencing the anastomotic healing play an important role in the outcome, once the fundamental principles of gastrointestinal suturing are followed. ${ }^{8}$ In this prospective study, we have evaluated the different variables known to cause anastomotic breakdown with an objective to identify the risk factors contributing to small intestinal anastomotic breakdown and also study the mortality and morbidity rates in patients undergoing small bowel anastomosis.

\section{METHODS}

All patients undergoing small bowel resection and anastomosis in our hospital, during the study period were considered for the study. Based on inclusion and exclusion criteria 74 patients were included in the study group and were divided into two groups, leak and non- leak group depending on occurrence of anastomotic leak. A prospective study was done on the risk factors known to influence the outcome of small bowel anastomosis were recorded in both groups and compared.

\section{Exclusion Criteria}

- Paediatric patients less than 12 years of age.

- Patients undergoing gastrointestinal, duodenal, colonic (including ileocolic and ileorectal) and rectal anastomosis,

- Patients who underwent intestinal stomal reversal.

- Patients who had undergone preoperative chemotherapy and radiotherapy.

Detailed history was taken and a thorough clinical examination done in all patients. Data was recorded on several factors which might contribute to anastomotic disruption. Patient's demographics (age, sex), details of surgery (emergency or elective, presence of peritonitis, indication for resection) and relevant investigations (haemoglobin, serum albumin, serum electrolytes, etc.) were all recorded and compared between the two groups. Haemoglobin of less than $10 \mathrm{~g} / \mathrm{dL}$ was defined as anaemia and serum albumin level of less than $3.5 \mathrm{~g} / \mathrm{dL}$ was defined as hypoalbuminemia. Serum sodium of less than $135 \mathrm{meq} / \mathrm{dL}$ was defined as hyponatremia. All patients undergoing emergency surgeries were adequately resuscitated before surgery. All the patients underwent double layer anastomosis, inner continuous suture to include all the layers and outer interrupted seromuscular sutures. All the patients received single dose of prophylactic antibiotic preoperatively and antibiotics were continued for 5-10 days based on diagnosis (presence or absence of peritoneal contamination).

\section{Statistical Analysis}

All the complications and mortality were recorded. Postoperative morbidity and mortality were defined as in hospital complications and death. Surgical site infection was diagnosed according CDC guidelines. Anastomotic leak was documented by reoperation, appearance of fistula or by imaging. Results were tabulated and statistically analyzed using Chi-Square test. ( $\mathrm{P}$ value of $<0.05$ was considered significant). 


\section{RESULTS}

\begin{tabular}{|c|c|}
\hline Indications & Percentage \\
\hline 1. Intestinal Obstruction-36 (48.6\%) & $48.6 \%$ \\
\hline • Strangulated Hernias- 23 & $31.08 \%$ \\
\hline Umbilical- 04 & $5.4 \%$ \\
\hline Inguinal- 17 & $22.9 \%$ \\
\hline Femoral - 02 & $2.7 \%$ \\
\hline - $\quad$ Intestinal strictures- 07 & $9.4 \%$ \\
\hline - $\quad$ Postoperative adhesions- 03 & $4 \%$ \\
\hline - $\quad$ Small bowel volvulus- 02 & $2.7 \%$ \\
\hline - Intussusception- 01 & $1.35 \%$ \\
\hline 2. Bowel perforation- $26(35.1 \%)$ & $35.1 \%$ \\
\hline - Typhoid perforation- 12 & $16.2 \%$ \\
\hline - $\quad$ Tubercular perforation- 04 & $5.4 \%$ \\
\hline - $\quad$ Others -10 & $13.5 \%$ \\
\hline 3. Tumours- 03 (2.7\%) & $2.7 \%$ \\
\hline 4. Others- $09(13.5 \%)$ & $13.5 \%$ \\
\hline - Acute mesenteric ischaemia- 05 & $6.7 \%$ \\
\hline - $\quad$ Meckel's diverticulum- 04 & $5.4 \%$ \\
\hline
\end{tabular}

\begin{tabular}{|cccc|}
\hline Sl. No. & Risk Factor & No. of Patients & Percentage \\
1 & Old age (60 yrs. or older) & 10 & $13.5 \%$ \\
2 & Male sex & 40 & $54 \%$ \\
3 & Anaemia & 14 & $18.9 \%$ \\
4 & Hypoalbuminemia & 12 & $16.2 \%$ \\
5 & Hyponatremia & 19 & $25.67 \%$ \\
6 & Peritonitis & 26 & $35.1 \%$ \\
7 & Emergency surgery & 67 & $90.5 \%$ \\
\hline \multicolumn{4}{|c|}{ Table 2. Incidence of Risk Factors in the Study } \\
\hline
\end{tabular}

\begin{tabular}{|ccc|}
\hline Sl. No. & Early Postoperative Complications & No. of Patients \\
1. & Surgical Site Infection & $21(28.3 \%)$ \\
2. & Respiratory complications & $14(18.9 \%)$ \\
3. & Anastomotic leak & $13(17.5 \%)$ \\
4. & Postoperative prolonged paralytic ileus & $4(5.4 \%)$ \\
5. & Pulmonary thromboembolism & $1(1.3 \%)$ \\
6. & Postop MI & $1(1.3 \%)$ \\
7. & Abdominal wound dehiscence (Burst abdomen) & $1(1.3 \%)$ \\
\hline \multicolumn{3}{|c}{ Table 3. List of Complications } \\
\hline
\end{tabular}

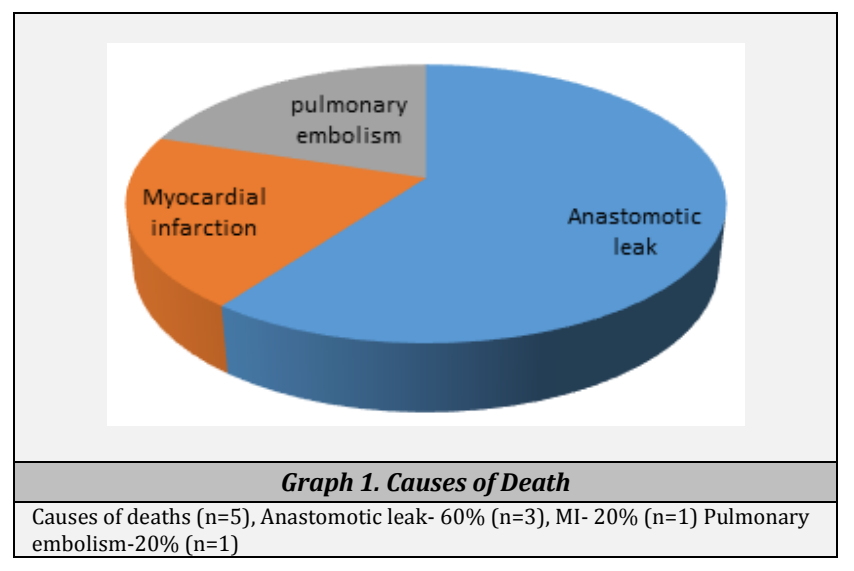

A total of 74 patients underwent small bowel anastomosis in our study period. Age of the patients ranged from 18 to 74 years. Indications for small bowel resection and anastomosis are given in Table 1. Intestinal obstruction was the most common indication (strangulated hernias being most common cause for obstruction) followed by small bowel perforation. Of 74 patients, 40 were males and 34 were females. There were 67 (90.5\%) emergency surgeries and 7 elective surgeries. 14 $(18.9 \%)$ patients were anaemic, $12(16.2 \%)$ patients were hypoalbuminaemic and 19 (25.6\%) were hyponatraemic on presentation. Peritonitis was present in 26 (35.1\%) patients. Table 2 shows the list of risk factors considered in this study with their incidence.

Among the study group 13 patients (17.5\%) developed anastomotic leak. The higher incidence of anastomotic breakdown is related to higher proportion of emergency surgeries $(90.5 \%)$ and peritoneal contamination $(35.1 \%)$ in the present study group. Of 13 patients, 5 patients presented with faecal fistula, 4 patients with features of peritonitis postoperatively, 3 patients with leakage of intestinal contents from drain site and 1 patients with postoperative fever and intra-abdominal abscess. CECT abdomen and pelvis was used to confirm the diagnosis in patient presenting with features of intra-abdominal abscess. Of 13 patients who developed anastomotic leak, 8 were males. One patient undergoing elective surgery developed anastomotic leak. Of 13 patients with anastomotic leak, 4 were anaemic, 4 were found to be hyponatraemic and 5 patients had hypoalbuminemia. Of 13 patients with anastomotic leak, 9 of them had peritonitis at presentation. Overall mortality rate was $6.7 \%$ (5 patients). Mortality rate in patients developing anastomotic leak was $23 \%$ (3 patients).

All deaths in the anastomotic leak group occurred due to septicaemia. Mortality in the non-leak group was $3.2 \%$ (2 patients), 1 patient developed postoperative MI and another developed DVT and pulmonary thromboembolism. Incidence of morbidity was $40.5 \%$ (30 patients). List of complications in the patients is shown in Table 3. Surgical site infection $(28.3 \%$ - 21 patients) was the most common surgical complication and respiratory complications (18.9\%-14 patients) were the most common medical complications in the present study. 5 patients with anastomotic leak were managed conservatively and remaining 8 underwent re-laparotomy and creation of stoma. After statistical analysis of the data, only hypoalbuminemia $(p=0.016)$ and presence of peritonitis $(p=0.004)$ were found to significantly increase the risk of anastomotic leak in the present study.

\section{DISCUSSION}

Anastomotic leak is the major cause of morbidity and mortality in patients undergoing bowel anastomosis. ${ }^{2}$ There is paucity of studies on understanding healing of small bowel anastomosis and risk factors contributing to anastomotic leak even though small bowel anastomosis is common procedure especially in the emergency setting. Anastomotic leak usually occurs usually between 3rd and 6th postoperative days. Patients with anastomotic leak may present with varied clinical features like faecal fistula, intra-abdominal abscess, persistent abdominal pain, fever, prolonged ileus, tachycardia, diarrhoea or features of intestinal obstruction.2,5 Diagnosis can be apparent clinically either by observing intestinal contents in drain or by presence of faecal fistula. ${ }^{2}$ When anastomotic leak is suspected clinically CT abdomen and pelvis with contrast may demonstrate intra-abdominal abscess or extravasation of contrast material. ${ }^{2}$ Reported rates of anastomotic leak in small bowel anastomosis ranges from $6.1 \%-35 \% \cdot{ }^{9-13}$ In this prospective study, we have evaluated the different risk factors responsible for anastomotic leak in small bowel anastomosis. The factors affecting gastro-intestinal healing can be divided into host related (age, sex, anaemia, hyponatremia, hypoalbuminemia, diabetes etc.), disease related (presence of contamination/malignancy etc.,) and those related to surgical technique (presence of tension along suture line, single vs. double layer, etc.). Following the basic principles of 
anastomotic surgery including creation of tension free anastomosis between well vascularized bowel segments with good approximation of edges and inversion of mucosa is essential part of small bowel anastomotic surgery. ${ }^{14}$ The submucosa provides the intact GI tract with most of its tensile strength and is responsible for anchoring the sutures that hold anastomosed bowel ends together. ${ }^{15}$ Hence, it is necessary to include submucosal layer in anastomosis especially when performing extra mucosal single layer anastomosis. ${ }^{15} \mathrm{~A}$ single layer intestinal anastomosis is as effective as double layer anastomosis and is also less time consuming and more cost effective. ${ }^{16}$ All the anastomosis in the present study are double layered, inner continuous layer including all the layers of intestine and outer layer of interrupted seromuscular sutures. With adequate supervision, there is little difference in outcome of anastomoses performed by trainees and those performed by established surgeons. ${ }^{8}$ Hence the host related factors are important determinants of outcome in anastomotic surgery, once the basic surgical principles of anastomotic surgery are followed. 8 Table 2 shows the list of factors influencing the outcome of anastomosis. Male sex, ${ }^{7}$ steroid use $^{7}$ anaemia, ${ }^{9,12}$ hypoalbunaemia, ${ }^{9,10}$ hyponatraemia, ${ }^{9}$ peritonitis, ${ }^{9,11-12}$ increased duration of surgery, ${ }^{9}$ intraoperative hypo-tension, $, 9,10$ and old age ( $>60$ years) ${ }^{17}$ have all been found to be associated with increased risk of anastomotic breakdown. Of all the variables studied, only presence of peritonitis and hypoalbuminemia were associated with increased risk of anastomotic leak in our study. 6 patients with hypoalbuminemia developed leak. 9 patients with peritoneal contamination developed anastomotic leak. Le Veen has postulated that the fibrinopurulent exudate filling the anastomotic space in presence of peritoneal sepsis prevents fibroplasia and angiogenesis from bridging the gap, as in healing by primary intention, and as a result anastomosis heals by secondary intention. ${ }^{18}$ Presence of intra-abdominal sepsis is shown to be reducing both collagen synthesis and mechanical strength in experimental colonic anastomotic healing. ${ }^{19}$

Hypoalbuminemia is an indicator of malnutrition, the mechanism through which malnutrition increases risk of anastomotic leak is not fully understood but could be due to lack of amino acids for collagen synthesis or impairment of innate immunity. Malnutrition has been shown to reduce the collagen content and its tensile strength in experimental colonic healing. ${ }^{20}$ Hypoalbunaemia is associated with impairment of macrophage activation and induce macrophage apoptosis, thereby impairing patient's immunity. ${ }^{21}$ Macrophages are important cells in the initial phases of anastomotic healing in gastro-intestinal tract. ${ }^{15}$

\section{CONCLUSIONS}

Presence of peritoneal contamination and hypoalbuminemia significantly increases the risk of anastomotic disruption in patients undergoing small bowel resection and anastomosis. In the presence of the above risk factors (especially if both risk factors are present), it is better to avoid primary anastomosis and consider these patients for creation of stoma to tide over the crisis followed by reversal of stoma once the patient's general condition and nutrition improves. In the elective setting, hypoalbuminemia and malnutrition can be corrected preoperatively to improve the outcome of anastomosis in these patients.

\section{REFERENCES}

[1] Williams NS, O'Connel RP, McCaskie AW. Bailey \& Love's Short practice of surgery. Chapter $-7.27^{\text {th }}$ edn. Taylor \& Francis Group, CRC Press 2018;1:97-8.

[2] Doherty GM. Current diagnosis \& treatment surgery. Chapter - 5. 14th edn. McGraw-Hill Education 2015.

[3] Fielding LP, Stewart-Brown S, Blesovsky L, et al. Anastomotic integrity after operations for large-bowel cancer: a multicentre study. $\mathrm{Br}$ Med J 1980;281(6237):411-4.

[4] Rahbari NN, Weitz J, Hohenberger W, et al. Definition and grading of anastomotic leakage following anterior resection of the rectum: a proposal by the International Study Group of Rectal Cancer. Surgery 2010;147(3):33951.

[5] Zinner MJ, Ashley SW. Maingot's Abdominal Operations. Chapter $-7.11^{\text {th }}$ edn. Tata McGraw-Hill Education 2007: p. 184-9.

[6] Edmunds LH, Williams GM, Welsh CE. External fistulas arising from the gastro-intestinal tract. Ann Surg 1960;152(3):445-71.

[7] Suding P, Jensen E, Abramson MA, et al. Definitive risk factors for anastomotic leaks in elective open colorectal resection. Arch Surg 2008;143(9):907-12.

[8] Hawkins WJ, Moorthy KM, Tighe D, et al. With adequate supervision, the grade of the operating surgeon is not a determinant of outcome for patients undergoing urgent colorectal surgery. Ann R Coll Surg Engl 2007;89(8):7605.

[9] Murugiah L, Mariappan K, Palani M. A study of risk factors influencing anastomotic leakage after small bowel anastomosis. J Evid Based Med Health 2017;4(40):241118.

[10] Nair A, Dinker RP, Jagadish S. Predicting anastomotic disruption after emergent small bowel surgery. Dig Surg 2006;23(1-2):38-43.

[11] Hesp WL, Lubbers EJ, De Boer HH. Anastomotic insufficiency in small bowel surgery - incidence and treatment. Langenbecks Arch Chir 1986;368(2):105-11.

[12] Al-Doghan I, Hussein HK. Leakage after small bowel anastomosis. Iraqi Med J 2015;61(1):23-9.

[13] Wegstapel H, Hoque HM, Hoile RW. Emergency smallbowel resection in a district general hospital. J R Soc Med 1998;91(12):638-9.

[14] Goligher JC, Morris C, McAdam WA, et al. A controlled trial of inverting versus everting intestinal suture in clinical large-bowel surgery. Br J Surg 1970;57(11):817-22.

[15] Thornton FJ, Barbul A. Healing in the gastrointestinal tract. Surgical Clinics of North America 1997;77(3):54973.

[16] Burch JM, Franciose RJ, Moore EE, et al. Single-layer continuous verses two-layer interrupted intestinal anastomoses: a prospective randomized trial. Ann Surg 2000;231(6):832-7.

[17] Irvin TT, Goligher JC. Aetiology of disruption of intestinal anastomoses. Br J Surg 1973;60(6):461-4. 
[18] LeVeen HH, Wapnick S, Falk G, et al. Effects of prophylactic antibiotics on colonic healing. Am J Surg 1976;131(1):4753.

[19] Ahrendt GM, Tantry US, Barbul A. Intra-abdominal sepsis impairs colonic reparative collagen synthesis. Am J Surg 1996;171(1):102-8.
[20] Irvin TT, Hunt TK. Effect of malnutrition on colonic healing. Ann Surg 1974;180(5):765-72.

[21] Rivadeneira DE, Grobmyer SR, Naama HA, et al. Malnutrition-induced macrophage apoptosis. Surgery 2001;129(5):617-25. 